\title{
Dynamic permeability in soft magnetic composite materials
}

A. Chevalier and M. Le Floc'h ${ }^{\text {a) }}$

Laboratoire d'Electronique et des Systèmes de Télécommunications (L.E.S.T.) UMR CNRS 6616, 6 avenue Le Gorgeu-BP 809-29285 Brest cedex, France

(Received 7 September 2000; accepted for publication 4 June 2001)

\begin{abstract}
This article reports on an isotropic model of the magnetic susceptibility based on an average field theory and proposes to predict the dynamic behaviors of powder magnetic materials. It was essentially built around a so-called effective demagnetizing factor capable of taking the particle shapes into account. So, for a population of randomly distributed particles of anisotropic shapes like, for instance, needles or flakes, we show that the effective demagnetizing factor of this population of particles can be significantly lowered with regard to the well known value of $1 / 3$ classically used to represent the isotropy state. This phenomenon is interpreted as the natural tendency of particles to form clusters to which a moving demagnetizing factor must be assigned. Taking then the aggregation process of particles into account, the ability of the model to predict the dynamic properties of many composite magnetic materials is successfully demonstrated. Our development is illustrated by experimental results concerning a nickel-zinc ferrimagnetic $\left(\mathrm{Ni}_{0.7} \mathrm{Zn}_{0.3} \mathrm{Fe}_{2} \mathrm{O}_{4}\right)$ powder. (C) 2001 American Institute of Physics. [DOI: 10.1063/1.1389520]
\end{abstract}

\section{INTRODUCTION}

Over the last years the study of composite materials aroused growing interest. We report here on microwave applications of composite materials consisting of soft magnetic particles embedded in insulating binders.

Several theories, reviewed by Greffe and Grosse, ${ }^{1}$ have been proposed to describe the dielectric properties of composite materials. One of the most known among them, the effective medium theory (EMT), has been developed by Bruggeman $^{2}$ and predicts the average susceptibility of inhomogeneous media. In a previous article ${ }^{3}$ we showed that, in the simple case of a two-phase mixture where only one of them was magnetic, the EMT leads to a simple and useful scalar expression as follows:

$$
(1-N) \chi^{2}+\left[1+(N-C) \chi_{i}\right] \chi-C \chi_{i}=0 .
$$

The magnetic susceptibility $\chi$ of the mixture depends on the intrinsic susceptibility $\chi_{i}$ of the magnetic substance as well as on the magnetic volume fraction $C$, and then on the so-called effective shape factor $N$ for the global representation of the microscopic demagnetizing effects. The next part of our study details how the factor $N$ can be connected to the shapes of particles and to their distributions in space.

\section{THEORETICAL DEVELOPMENT}

In order to simply calculate the effective factor $N$, we assume that the particles of concern can be replaced by ellipsoids whose the associated triplet of demagnetizing constants $\left(N_{x}, N_{y}, N_{z}\right)$ is fixed by the ratios of the respective lengths of their semi-axes. One can, then, calculate the diagonal tensor ${ }^{4}$ of the internal field, $H_{i}$, for such ellipsoid particles of permeability, $\mu_{i}$, embedded in a homogeneous medium of permeability, $\mu$ (Fig. 1).

\footnotetext{
${ }^{a)}$ Electronic mail: marcel.lefloch@univ-brest.fr
}

The isotropic character of the mixture is, next, introduced through the assumption of the random distribution of particles in space. The mean internal field $\left\langle H_{i}\right\rangle$ is derived from the average of the three earlier components

$$
\left\langle H_{i}\right\rangle=\frac{1}{3}\left(H_{i / x}+H_{i / y}+H_{i / z}\right) .
$$

Since the demagnetizing factors satisfy the conditions: $N_{x}+N_{y}+N_{z}=1$ and $N_{x}=N_{y}$ then, effective data on particle shapes are reduced to the only parameter $N_{z}$. The internal field $\left\langle H_{i}\right\rangle$, then, becomes

$$
\left\langle H_{i}\right\rangle=\left[\frac{2}{\mu+\left(\mu_{i}-\mu\right)\left(1-N_{z}\right) / 2}+\frac{1}{\mu+\left(\mu_{i}-\mu\right) N_{z}}\right] \frac{\mu H}{3} .
$$

In the meaning of the effective medium theory applied to the isotropic case, we choose to define ${ }^{5,6}$ the effective shape factor $N$ by taking for $\left\langle H_{i}\right\rangle$ the same formal expression as that of the $H_{i}$ components

$$
\left\langle H_{i}\right\rangle=\frac{H}{1+\left(\mu_{i}-\mu / \mu\right) N} .
$$

The equality between relations (3) and (4), then, allows us to connect $N$, first to the real parameter of shape $N_{z}$ of the magnetic particles, and second to the volume fraction $C$. The effective shape factor $N$ is then given by

$$
N=\frac{2+3 \delta N_{z}\left(1-N_{z}\right)}{6+\delta+3 \delta N_{z}},
$$

where $\delta=\chi_{i} /(1+\chi)$ is a so-called factor of contrast, which itself depends on the volume fraction $C$.

The values of $N$ are calculated by solving Eqs. (1) and (5) together. Figure 2 shows that the effective shape factor $N$ depends both on the magnetic load $C$ and on the real shape factor $N_{z}$ of particles. For separated spherical particles $N$ is equal to $1 / 3$ over the whole volume fraction range. On the other hand, in all other cases, i.e., needle- or flake-type par- 


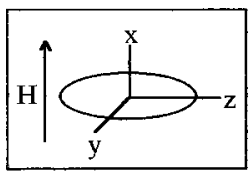

$H_{i / x}=\frac{H}{1+\frac{\mu_{i}-\mu}{\mu} N_{x}}=\frac{\mu H}{\mu\left(\mu_{i}-\mu\right) N_{x}}$

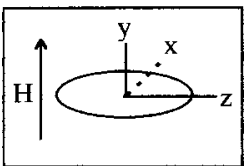

$\mathrm{H}_{\mathrm{i} / \mathrm{y}}=\frac{\mathrm{H}}{1+\frac{\mu_{\mathrm{i}}-\mu}{\mu} \mathrm{N}_{\mathrm{y}}}=\frac{\mu \mathrm{H}}{\mu\left(\mu_{\mathrm{i}}-\mu\right) \mathrm{N}_{\mathrm{y}}}$

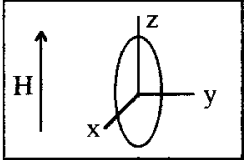

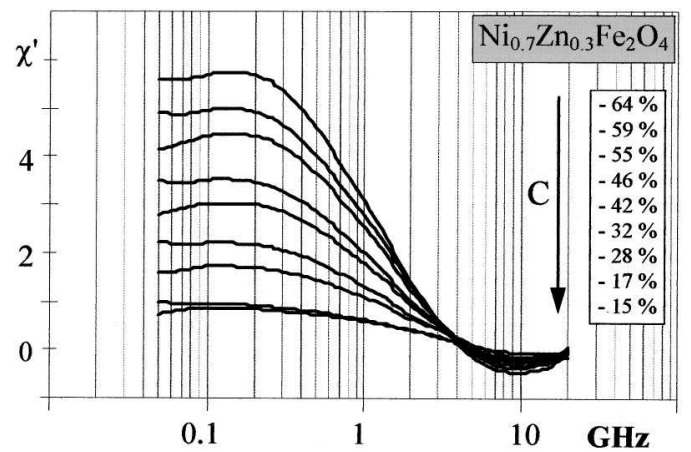

FIG. 3. Experimental variations of the real part $\chi^{\prime}$ of susceptibility of $\mathrm{Ni}_{0.7} \mathrm{Zn}_{0.3} \mathrm{Fe}_{2} \mathrm{O}_{4}$ magnetic composites.

magnetizing fields. The magnetic volume fraction ranged within a few percent to about $60 \%$. Thermal densifications were not used in this study.

The initial dynamic susceptibility of the composite material $\left[\chi(\omega)=\chi^{\prime}(\omega)-j \chi^{\prime \prime}(\omega)\right]$ was investigated at room temperature in the frequency range $50 \mathrm{MHz}-20 \mathrm{GHz}$ by using a Hewlett-Packard APC7 coaxial-line technique and network analyzers. The results are shown in Figs. 3 (real parts) and 4 (imaginary parts) for different magnetic fractions in the earlier mentioned range. The reduction of the magnetic load lowers the magnitude of the susceptibility. This behavior is general and usually expected.

However, as the magnetic resonance frequency (MRF) is spotted by the maximum of the imaginary part of susceptibility, its behavior is clearly different: first, it remains unchanged, then increases continuously below a certain threshold $C_{p}$ close to $30 \%$.

We consider this resonance frequency shift as being a direct consequence of the increase of the microscopic demagnetizing fields in the mixture in connection with the modifications of dipolar-type interactions on the one hand, and of the particle arrangements on the other hand. This is quite consistent with other studies which have also clearly highlighted the basic role of the demagnetizing effects ${ }^{8}$ on the dynamic properties of the magnetic susceptibility in composite materials. As the volume fraction decreases, the local demagnetizing fields add to the total anisotropy field and consequently increase the resonance frequency. In the next

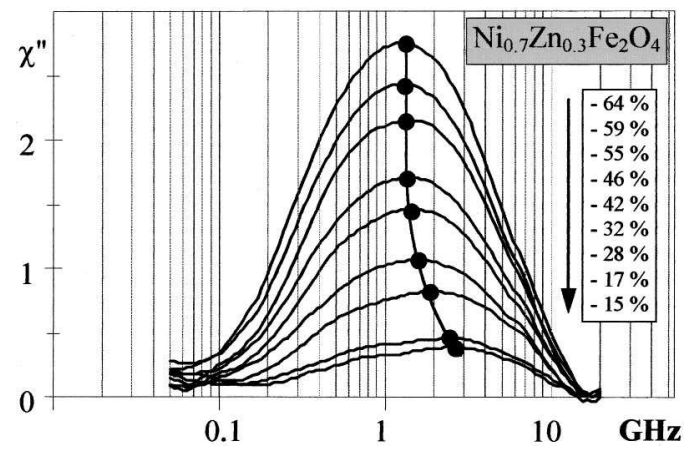

FIG. 4. Experimental variations of the imaginary part $\chi^{\prime \prime}$ of susceptibility of $\mathrm{Ni}_{0.7} \mathrm{Zn}_{0.3} \mathrm{Fe}_{2} \mathrm{O}_{4}$ magnetic composites. Full circles $(\mathbf{O})$ spot the resonance frequency shift.
FIG. 2. Effective shape factor $N$ as a function of the magnetic load $C$ and of the particle shape factor $\left(N_{z}\right)$. Theoretical values are obtained by solving the couple of Eqs. (1) and (5) for $\chi_{i}=20$. 


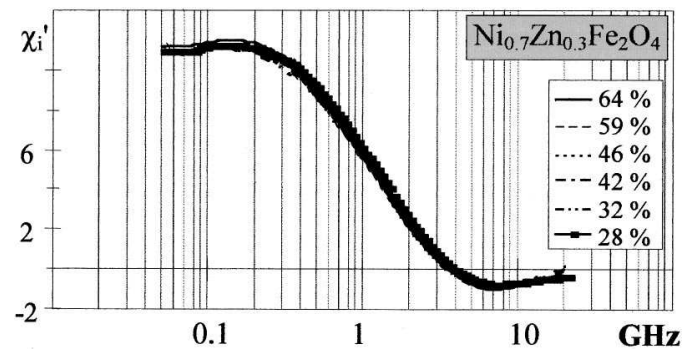

FIG. 5. Real part of the dynamic intrinsic susceptibility $\chi_{i}^{\prime}$ obtained with the inversion of experimental data of Fig. 3.

section we will demonstrate that the model proposed in this study takes properly this internal effect into account.

\section{COMPARISONS WITH THE MODEL}

To process experimental data in order to extract the intrinsic susceptibility of the magnetic matter, we use Eq. (1) in which $\chi_{i}$ is taken as the unknown variable, whereas $C$ and $\chi$ come from measurements. $N$ is then adjusted under the obvious condition that it must remain less than or equal to $1 / 3$. This leads to the noteworthy transformation of Figs. 3 and 4 into the reduced curves of Figs. 5 and 6. One should, indeed, note that, over the whole frequency range and for the different magnetic fractions, there is a unique solution for $N$; in consequence, the model we use [see Eq. (1)] can describe both the decreasing magnitude of the susceptibility and the shift in the MRF observable in composites magnetic materials.

Figure 7 shows the experimental values of the earlier solution for $N$. They correspond to the expected intrinsic susceptibility and are absolutely consistent with the theoretical curves predicted in Fig. 2. They are not only less than $1 / 3$, but they also depend on the volume fraction.

\section{DISCUSSION}

We showed in the previous section that the effective shape factor $N$ depends on both the real particle shape $\left(N_{z}\right)$ and the magnetic fraction $C$ [Eq. (5)]. Thus, theoretically in the case of spherical particles, the effective shape factor $N$ must be equal to $1 / 3$ and not depend on the magnetic fraction (see Fig. 2). However, experimental values are systematically below $1 / 3$ and magnetic fraction-dependent even in the case of spherical magnetic particles (Fig. 7). This discrepancy, in fact, results from the unavoidable aggregations of

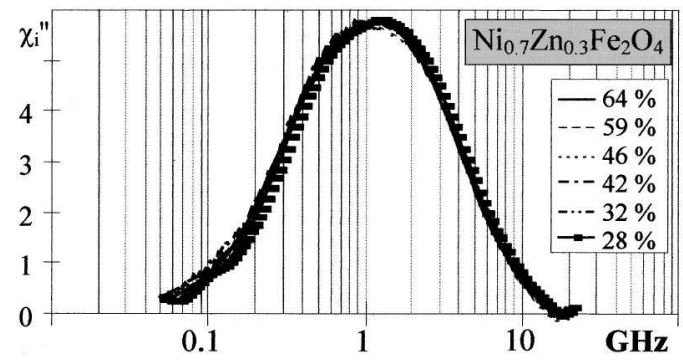

FIG. 6. Imaginary part of the dynamic intrinsic susceptibility $\chi_{i}^{\prime \prime}$, obtained with the inversion of experimental data of Fig. 4.

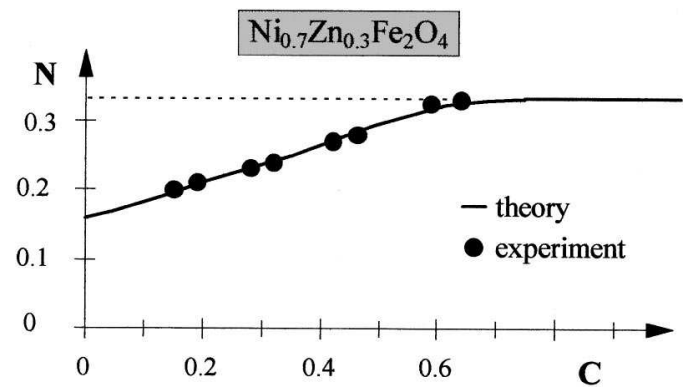

FIG. 7. Experimental and theoretical values of the effective shape factor $N$ obtained to superimpose dynamic spectra of Figs. 5 and 6 .

particles during the powder mixing processes, particularly when they are of very small sizes. By way of consequence the clusters are perceived as new particles distorted and bigger than the others. It is then obvious that the shape distribution of such aggregates cannot be evaluated by direct (microscopy) observations, especially because they are sensitive to the changes in the magnetic load. This is, in fact, quite a general problem which largely goes beyond the limits of this study.

It is, however, quite possible from simple and practical assumptions to build an intuitive function of distribution of the cluster shapes. First, any aggregation of individual ellipsoidal particles necessarily results in clusters of ellipsoidal shapes with a demagnetizing factor $N_{z}$ (see Fig. 8). Second, the probability $P\left(N_{z}\right)$ to find clusters with a given shape depends on the magnetic fraction as follows: at low concentrations, the particles tend to remain linked in small chainlike aggregates so as they are equivalent to prolate ellipsoids, ${ }^{9}$ and then the composite becomes equivalent to a mixture of needle-type particles for which $N_{z}$ is by definition less than $1 / 3$. On the contrary, at high concentrations, the clusters grow in any direction and progressively tend to become spherical: by definition, $N_{z}$ is here equal to $1 / 3$. In our study we considered that the probability to develop flake-like aggregates for which $N_{z}$ is by definition higher than $1 / 3$ was unrealistic.

\begin{tabular}{|c|l|c|c|}
\hline cluster & associated ellipsoid & $\mathrm{c} / \mathrm{a}$ & $\mathrm{N}_{\mathrm{z}}$ \\
\hline $\mathrm{O}$ & $\left.\mathrm{b}\right|^{\mathrm{a}} \mathrm{1}$ & $1 / 3$ \\
\hline 0 & $\left.\mathrm{~b}\right|^{\mathrm{a}} \mathrm{2}$ & 0.173 \\
\hline 00 & $\left.\mathrm{~b}\right|^{\mathrm{a}}$ & 3 & 0.108 \\
\hline 830 & $\left.\mathrm{~b}\right|^{\mathrm{a}}$ & 2.5 & 0.089 \\
\hline 800 & $\mathrm{~b}$ & 0.3 & 0.661 \\
\hline
\end{tabular}

FIG. 8. Identification of aggregates with ellipsoid. 


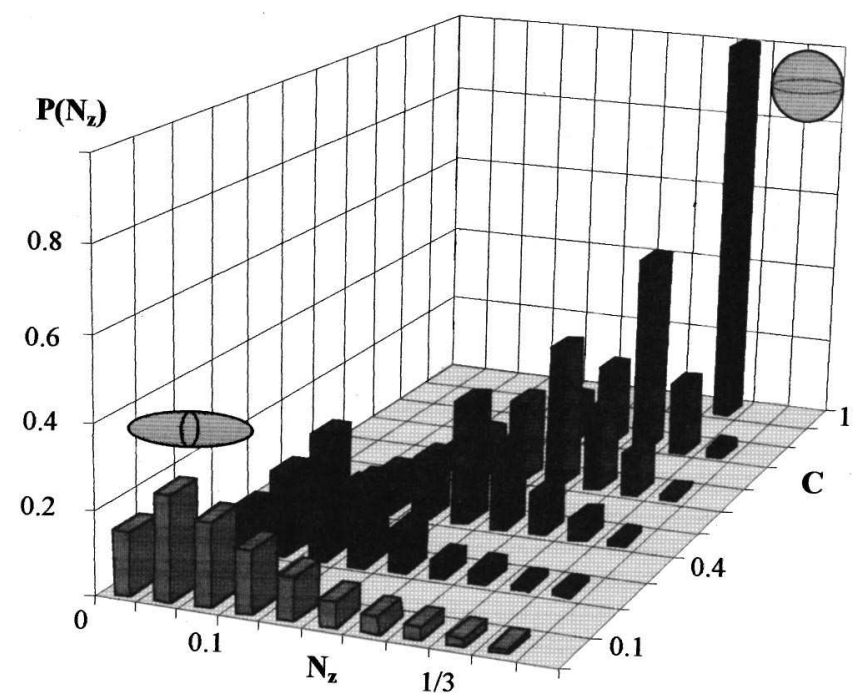

FIG. 9. Statistical distribution $P\left(N_{z}\right)$ of aggregates shape $\left(N_{z}\right)$ with the volume fraction $C$.

The earlier assumptions are sufficient to design a convincing profile of the distribution law $P\left(N_{z}\right)$. Indeed, as the magnetic concentration increases, the function $P\left(N_{z}\right)$ must have a maximum whose position is shifted towards the high values of $N_{z}$ up to $1 / 3$ (strictly speaking) when the magnetic fraction reaches unity. Simultaneously, the profile of $P\left(N_{z}\right)$ must become narrower and narrower. Figure 9, finally, shows the histogram of $P\left(N_{z}\right)$ which was used to realize the very satisfactory fit of Fig. 7.

For an exact computation of the effective shape factor $N$, Eq. (3) can be now easily expressed as a continuous sum acting on $N_{z}$ as follows:

$$
\begin{aligned}
\left\langle H_{i}\right\rangle= & \int_{0}^{1}\left[\frac{2}{\mu+\left(\mu_{i}-\mu\right)\left(1-N_{z}\right) / 2}\right. \\
& \left.+\frac{1}{\mu+\left(\mu_{i}-\mu\right) N_{z}}\right] \frac{\mu H}{3} P\left(N_{z}\right) d N_{z} .
\end{aligned}
$$

When the particle concentration in a given mixture is increasing, aggregates are rapidly formed; their shapes are, then, continuously changing from chains to spheres up to the concentration unity where the medium becomes isotropic. A further work would be in deriving a mathematical function of the distribution $P\left(N_{z}\right)$ to have finally a completely predicting model. This is an interesting challenge because it is known that the dynamic properties of magnetic powder materials are largely influenced by such a term.

\section{CONCLUSION}

An extension of the effective medium theory was proposed to predict the dynamic behaviors of isotropic composite materials. This model is capable of describing experimental results, namely the variation of magnitude of permeability, together with the shift of the resonance frequency. While taking into account the formation of particle aggregates, we derived the intrinsic permeability of magnetic matter independently of the magnetic fraction of the mixture. An immediate and interesting extension of this work would be the determination of an analytical function of the magnetic fraction of the mixture to take the grain structure contribution into account.

${ }^{1}$ J. L. Greffe and Grosse, Dielectric Properties of Heterogeneous Materials, P.I.E.R. 6 (Elsevier, New York, 1992).

${ }^{2}$ D. A. G. Bruggeman, Ann Phys. (Leipzig) 24 (1935).

${ }^{3}$ M. Le Floc'h, J. L. Mattei, P. Laurent, O. Minot, and A. M. Konn, J. Magn. Magn. Mater. 140 (1995).

${ }^{4}$ J. A. Stratton, Electromagnetic Theory (McGraw-Hill, New York, 1941).

${ }^{5}$ M. Le Floc'h, A. Chevalier, and J. L. Mattei, J. Phys. IV 8, 2 (1998).

${ }^{6}$ A. Chevalier, Ph.D. thesis, Université de Bretagne Occidentale, 1998.

${ }^{7}$ T. Takada and M. Kiyama, Proc. Of Intern. Conf., Japan 1970, p. 69.

${ }^{8}$ C. Kittel, Phys. Rev. 73, 155 (1948).

${ }^{9}$ C. G. Granqvist and O. Hunderi, Phys. Rev. B 18, 1554 (1978). 